\title{
Integrated System Dramatically Improves Hydrogen Molar Yield from Biomass via Fermentation
}

Project: Fermentative and Electrohydrogenic Production of Hydrogen

Team: Pin-Ching Maness and Shiv Thammannagowda, NREL; Bruce Logan, Pennsylvania State University

Accomplishment: NREL and Penn State University developed a novel integrated system that improves hydrogen molar yield via fermentation by a factor of four to eight, eclipsing the U.S. Department of Energy's (DOE's) 2013 target (first reported in February 2009). The system uses dark fermentation of lignocellulosic corn stover followed by a microbial electrolysis cell (MEC) reaction. NREL's fermentation process employs an easily scalable and fast conversion of recalcitrant biomass to hydrogen along with waste byproducts. The fermentation effluent is used in an MEC to produce additional hydrogen.

NREL's work on fermentative hydrogen production from lignocellulosic biomass, led by Pin-Ching Maness, focuses on a cellulose-degrading thermophilic bacterium, Clostridium thermocellum. This microbe is reported to have one of the highest rates of cellulose degradation, converting the recalcitrant yet abundant corn stover lignocellulose directly to hydrogen; the hydrogen molar yield is 1.67 mol hydrogen per mol hexose in a scale-up bioreactor. Researchers at Penn State, led by Bruce Logan, optimized the MEC process and adapted microbes immobilized on the anode to oxidize the waste organic acids and ethanol present in the fermentation effluent. The MEC cathode efficiently used the extracted electrons to produce hydrogen. The integrated process results in a combined hydrogen molar yield greater than 8.7 from corn stover lignocellulose, capturing approximately $73 \%$ of the energy in cellulose as hydrogen.

Context: The high cost of glucose feedstock and the low hydrogen molar yield have hindered the applications of fermentative hydrogen production. Most laboratories report a hydrogen molar yield of 1 to 2 using glucose as the substrate. The NREL-Penn State results improve hydrogen molar yield by four- to eight-fold using lignocellulose, in lieu of glucose, as the substrate.

Applicable DOE Technical Target: DOE 2013 target for dark fermentative hydrogen production (achieve 4 molar yield of hydrogen from glucose).

Significance of Accomplishment: The combined hydrogen molar yield addresses DOE technical barriers and improves the economic feasibility of hydrogen production via fermentation. NREL's findings show that it is possible to obtain a hydrogen molar yield of 8.7 from pretreated biomass (dilute acid hydrolysis of corn stover) via integration of the two subsystems. These results show a molar yield that exceeds DOE's 2013 target. A preliminary NREL boundary analysis revealed that with a hydrogen molar yield of 8 and a glucose selling price of $\$ 0.10 / \mathrm{lb}$, a hydrogen selling price of $\$ 2.50 / \mathrm{kg}$ (based on feedstock cost only) can be realized. Now an analysis is being conducted to calculate the cost of using lignocellulose from corn stover as a feedstock to produce hydrogen using this integrated system.

\begin{tabular}{|c|c|c|}
\hline \multicolumn{3}{|c|}{$\begin{array}{c}\text { Hydrogen Production in the Fermentation-MEC } \\
\text { Integrated System }\end{array}$} \\
\hline & $\begin{array}{l}\text { Corn Stover } \\
\text { Biomass Pretreated } \\
\text { with Dilute Acid }\end{array}$ & Cellobiose* \\
\hline $\begin{array}{l}\mathrm{H}_{2} \text { Molar Yield via } \\
\text { Fermentation }\end{array}$ & 1.67 & 1.64 \\
\hline $\begin{array}{l}\mathrm{H}_{2} \text { Molar Yield via } \\
\text { MEC }\end{array}$ & $>7$ & 8.31 \\
\hline $\begin{array}{l}\text { TOTAL } \mathrm{H}_{2} \text { Molar } \\
\text { Yield }\end{array}$ & $>8.7$ & 9.95 \\
\hline \multicolumn{3}{|c|}{$\begin{array}{l}\text { *The bacterium breaks down cellulose to cellobiose and ferments the latter t } \\
\text { hydrogen. Cellobiose data are included to show that the bacterium performs } \\
\text { equally well using either the real complex substrate (lignocellulose) or the } \\
\text { pure, simple yet costly sugar. }\end{array}$} \\
\hline
\end{tabular}

